\title{
Dynamics of the twisted magnetic flux tube in the astrophysical jets
}

\section{Ebru Devlen}

Ege University, Science Faculty, Department of Astronomy and Space Sciences, 35100, Bornova, Izmir, TURKEY

E-mail: ebru.devlen@ege.edu.tr

We investigate the local linear stability analysis of rotating jets under the thick flux-tube approximation. The magnetic field is chosen so as to have toroidal and poloidal components contributing to the internal twist of the flux-tube. We obtained the dispersion relation for structures of the binormal helices. We also did numerical solutions of the dispersion relation. In the smaller $m$ modes, the stabilizing effect of the rigid rotation is remarkable, since rotation velocities of the higher than the Alfven speed fully stabilize the flow in many cases. However, it is clearly seen that the higher $m$ modes are generally more stable.

VII Microquasar Workshop: Microquasars and Beyond

Foca, Izmir, Turkey

September $1^{\text {st }}-5^{\text {th }} 2008$ 


\section{Introduction}

Today, collimated MHD outflows are analytically investigated in two different approaches (Vlahakis \& Tsinganos, 1998): 1) RSSM, that, is the radially self-similar models, the prototype of which is the Blandford \& Payne (1982) model and 2) Sauty \& Tsinganos (1994) model which is also known as the MSSM - meridionally self-similar model. Solar/stellar winds may be regarded as the evolved jets after the sun/stars lose angular momentum (ST94, STT99, STT02). But, the numerical simulations carried out by Tsinganos \& Bogovalov (2002) and Matt et al. (2003) showed that magnetocentrifugally collimated outflows from a rotating celestial object or Keplerian accretion discs contain rather less mass and magnetic flux than the surrounding winds. The same conclusion is reached in ST94 and STT02. ST94 claims that the greater part of the mass loss rate comes from the disc. However, observations points to the contrary to these studies' predictions, i.e., collimated outflows bear higher fluxes. To get out of this dilemma, ST94, Koide et al. (1998), STT02, and Tsinganos \& Bogovalov (2002) proposed that the jets may be regarded as a two component system: an outflow coming from the central object and a disk wind. Hartigan et al (1995) showed that the jets have shown two velocity components, one of which (LVC) has a velocity range of $10-50 \mathrm{~km} / \mathrm{s}$ and the other (HCV) has a radial velocity of several hundred $\mathrm{km} / \mathrm{s}$. Kwan \& Tademaru (1995) proposed that LVC is the disc wind enveloping the jet and this proposition was confirmed by Bacciotti et al. (2002) with their study on DG Tau jet the data of which was taken by HST/STI. The kinematics of the jet revealed an onion like shape with HVC lying close to the axis and LVC surrounding it.

The above mentioned two component jets are also revealed by time dependent simulations of jets emanating from black holes and stars (e.g. Kudoh et al., 1998, Koide et al., 1998). Inner plasma of the $\mathrm{BH}$ is compressed and the pressure as an outward driving force develops. Enveloping wind is driven out centrifugally and collimated by the gradient of the azimuthal component of the magnetic field. But, the double jet component simulations and the analytical solutions enjoy a partial success, that is, while the former can give an account to dense core jets, the latter can better explain the hollow jets. The common conclusion is reached by both the models on the flow stability, that is the plasma is more stable at the inner flow than the Keplerian outer part of the hollow jets (Hanasz et al., 2000; Kudoh et al., 2002).

Hanasz et al (2000) showed that a local analysis on the thin flux tube is more stable to the magnetorotational instability than the enveloping jet originating from the jet. They also showed that in some cases rotation stabilizes the disc completely.

Now, what may be called our contribution to this field may be outlined as below. Our analysis is partly, not wholly, an extension of the study by Hanasz et al. (2000), wherein the linear stability of rotating jets confined by a toroidal magnetic field is analyzed by using thin flux tube approximation. They find that if the azimuthal velocity is of the order of or higher than the Alfven azimuthal speed, the rigidly rotating part of the jet interior can be completely stabilized, while the strong shearing instability operates in the transition layer between the rotating jet interior and the external medium. This can explain the limb-brightening effect observed in several jets. However, it is still possible to find jet equilibria that are stable all across the jet, even in the presence of differential rotation.

In this study to examine the stability problem of the rotating jets we have used thick fluxtube approximation because we considered curvature and torsion effects and twist angle. The magnetic field is chosen so as to have toroidal and poloidal components contributing to the internal twist of the flux-tube. The plan of the paper is as follows. Section 2 equations of flux tube dynamics are considered. In section 3 fundamental equations for the linear analysis of the stability are addressed. In section 4 numerical solutions of the dimensionless dispersion relation are given. Finally, in section 5 we mentioned future studies. 


\section{Equations of flux tube dynamics}

We use Ricca's Riemann geometrical model for the twisted flux tube (Ricca 2005). For the sake of simplicity we consider a cylindrical, nonrelativistic jet in cylindrical coordinates $(R, \phi, z)$ with a poloidal and toroidal magnetic field, $\mathbf{B}_{p}=\left[0, B_{\theta}(r, \theta(s), 0)\right]$ and $\mathbf{B}_{t}=\left[0,0, B_{t}(r)\right]$, assuming that the radial (expansion) velocity vanish. The longitudinal velocity $V_{z}$ can also be eliminated through a transformation to the reference frame comoving with the jet. Such a rotating equilibrium satisfies the transfield equation (see e.g. Chan \& Henriksen 1980)

$$
\frac{d p_{g}}{d r}-\rho \frac{V_{\varphi}^{2}}{r}+\frac{d}{d r}\left(\frac{B_{\varphi}^{2}}{8 \pi}\right)+\frac{1}{r} \frac{B_{\varphi}^{2}}{4 \pi}+\frac{B_{z}}{4 \pi} \frac{d B_{z}}{d r}=0
$$

In the Lagrangian approach the equations describing the flux tube dynamics in the thick flux tube approximation are given by the following:

i) The equation expressing the magnetohydrostatic pressure balance of the internal (subscript i) gas and magnetic pressure of the flux tube with the total pressure of the external medium (subscript e),

$$
p_{i}+\frac{B_{i}(s, t)^{2}}{8 \pi}=p_{e}
$$

ii) The flux freezing conditon following from the induction and continuity equations,

$$
\frac{D}{D t}\left(\frac{\mathbf{B}}{\rho}\right)=\left(\frac{\mathbf{B}}{\rho} \cdot \nabla\right) \mathbf{v}
$$

iii) The polytropic equation of state,

$$
p_{i} \rho_{i}^{-\gamma}=\mathrm{sbt}
$$

iv) The equation of motion,

$$
\rho_{i} \frac{D \mathbf{v}_{i}}{D t}=-\frac{1}{K} \frac{\partial p_{T}}{\partial s} \mathbf{e}_{t}+\rho_{e}\left(\mathbf{v}_{e} \cdot \nabla\right) \mathbf{v}_{e}+\frac{(\mathbf{B} \cdot \nabla) \mathbf{B}}{4 \pi}-\rho_{i} \mathbf{\Omega} \times(\boldsymbol{\Omega} \times \mathbf{r})+2 \rho_{i} \mathbf{v}_{i} \times \mathbf{\Omega}
$$

The gradient of the total pressure in (5) can be eliminated using equation which describes the stationary equilibrium of the external medium. By projecting on the generalized Frenet basis we obtain the equations for the motion of a thick magnetic flux tube.

\section{Linear stability analysis}

In this section we calculate the geometrical changes caused by a small displacement which we apply to the equilibrium pathof the tube in order to determine its stability properties. We perturb the flux tube by a Lagrangian displacement, $\xi$, of its equilibrium path, $\mathbf{r}_{0}\left(s_{0}\right)$, such that the 
position of a fluid element which initally was at arc length $s_{0}$ is given by $\mathbf{r}=\mathbf{r}_{0}\left(s_{0}\right)+\boldsymbol{\xi}\left(s_{0}, t\right)$. We express $\xi$ in terms of the generalized Frenet basis $\left(\mathbf{e}_{r}, \mathbf{e}_{\theta}, \mathbf{e}_{t}\right)$ and the cylindirical polar basis:

$\xi\left(s_{0}, t\right)=\xi_{r} \mathbf{e}_{r 0}+\xi_{\theta} \mathbf{e}_{\theta 0}+\xi_{t} \mathbf{e}_{t 0}$

$\xi\left(s_{0}, t\right)=\xi_{R} \mathbf{e}_{R 0}+\xi_{\phi} \mathbf{e}_{\phi 0}+\xi_{z} \mathbf{e}_{z 0}$

The relation between the bases in the unperturbed configuration for binormal helix structure is

$\mathbf{e}_{t 0}=\mathbf{e}_{\phi 0} ; \quad \mathbf{e}_{n 0}=\mathbf{e}_{r 0}=-\mathbf{e}_{R 0} ; \quad \mathbf{e}_{b 0}=\mathbf{e}_{\theta 0}=\mathbf{e}_{z 0}$

$\frac{\partial \mathbf{e}_{t 0}}{\partial s_{0}}=\kappa_{o} \mathbf{e}_{r 0} ; \quad \frac{\partial \mathbf{e}_{r 0}}{\partial s_{0}}=-\kappa_{o} \mathbf{e}_{t 0}+\tau_{0} \mathbf{e}_{\theta 0} ; \quad \frac{\partial \mathbf{e}_{\theta 0}}{\partial s_{0}}=-\tau_{0} \mathbf{e}_{r 0}$

For the present case of a flux-tube $\left(s_{0}=r_{0} \phi_{0}\right)$, we convert all spatial derivatives with respect to $s_{0}$ into partial derivatives with respect to the unperturbed azimuthal coordinate, i.e. $\frac{\partial}{\partial s_{0}}=\frac{1}{r_{0}} \frac{\partial}{\partial \phi_{0}}$. Consequently, solution can be written in the form $\xi=|\xi| \exp \left(i m \phi_{0}-i \omega t\right)$ where $|\xi|$ is the amplitude, $\omega$ the frequency and $m$ the (integer) azimuthal wavenumber. Thus we have $\frac{\partial}{\partial \phi_{0}} \rightarrow i m, \frac{\partial}{\partial t} \rightarrow-i \omega$.

The resulting dimensionless dispersion relation is given below:

$$
a_{4} \tilde{\omega}^{4}+a_{3} \tilde{\omega}^{3}+a_{2} \tilde{\omega}^{2}+a_{1} \tilde{\omega}+a_{0}=0
$$

\section{Numerical solution of the dispersion relation}

To study the instability in different ranges of the multidimensional parameter space we will present imaginary part of the growth rate of the instability versus the azimuthal Mach number and the magnitude shear force, for different choices of the other parameters. Figure 1 show the instability growth rate at a fixed radius $r=r_{0}=1$, for different modes $m=1,3$ and different plasma beta value $\beta=0.1,10$. The Alfven speed $v_{A}=1$ and the density at $r_{0}, \rho_{0}=1$ are fixed.

The Figure 1 shows that the configuration is strongly destabilized by the shear force in all cases. In the smaller $m$ modes, the stabilizing effect of the rigid rotation is remarkable, since rotation velocities of the higher than the Alfven speed fully stabilize the flow in many cases. However, it is clearly seen that the higher $m$ modes are generally more stable. This is because the higher $m$ modes correspond to greater bending and thus exposed to more of the restoring magnetic tension. 


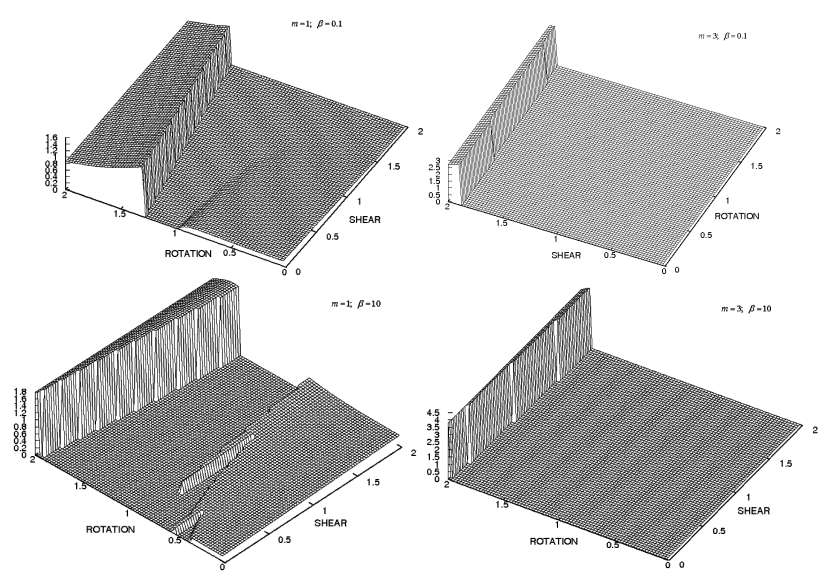

Fig. 1: The growth rate for the $\beta=0.1$ and $10, m=1$ and 3 mode versus rotation and shear with all the forces taken into account.

\section{Future studies}

Firstly, we will check the dispersion relation, becuse the coefficents of the dispersion relation is so long. Secondly, we'll solve dispersion relation for another parameters and also different range. We'll consider several idealized jet radial equilibria in order to illusturate consequences of our results for jet models. Lastly, we compare our results with theoritical and observational arguments.

\section{References}

[1] Tsinganos K., Bogovalov S., 2002, MNRAS, 337.

[2] Blandford R. D., Payne D. G., 1982, MNRAS, 199, 883.

[3] Sauty C., Tsinganos K., 1994, A\&A, 287, 893 (ST94).

[4] Sauty C., Tsinganos K., Trussoni E., 1999, A\&A, 348, 327 (STT99).

[5] Sauty C., Tsinganos K., Trussoni E., 2002, A\&A, 389, 1068 (STT02).

[6] Matt S., Winglee R., B"ohm K-H., 2003, MNRAS, 345, 660.

[7] Koide S., Shibata K., Kudoh T., 1998, ApJ, 508, 186.

[8] Hartigan P., Edwards S., Ghandour L., 1995, ApJ, 452, 736.

[9] Kwan J., Tademaru E., 1995, ApJ, 454, 382.

[10] Bacciotti F., Ray T.P., Mundt R., Eisloffel J., Solf J., 2002, ApJ, 576, 222.

[11] Kudoh T., Matsumoto R., Shibata K., 1998, ApJ, 508, 186.

[12] Hanasz M., Sol H., Sauty C., 2000, MNRAS 316, 494.

[13] Kudoh T., Matsumoto R., Shibata K., 2002, PASJ, 54, 121.

[14] Ricca R. L., 2005, Fluid Dynamic Res., 36, 319.

[15] Chan K. L., Henricksen R. N., 1980, ApJ, 241, 534. 